Archives de sciences sociales des religions

131-132 | juillet - décembre 2005

Varia

\title{
L'Église orthodoxe de Grèce et le « combat » des cartes d'identité (2000-2001)
}

Isabelle Dépret

\section{OpenEdition}

1 Journals

Édition électronique

URL : http://journals.openedition.org/assr/3254

DOI : 10.4000/assr.3254

ISSN : $1777-5825$

Éditeur

Éditions de l'EHESS

Édition imprimée

Date de publication : 1 décembre 2005

Pagination : 27-46

ISBN : 2-7132-2045-9

ISSN : 0335-5985

Référence électronique

Isabelle Dépret, «L'Église orthodoxe de Grèce et le « combat » des cartes d'identité (2000-2001) », Archives de sciences sociales des religions [En ligne], 131-132। juillet - décembre 2005, mis en ligne le

30 juin 2008, consulté le 19 avril 2019. URL : http://journals.openedition.org/assr/3254 ; DOI :

10.4000/assr.3254

Ce document a été généré automatiquement le 19 avril 2019.

(c) Archives de sciences sociales des religions 


\title{
L'Église orthodoxe de Grèce et le « combat » des cartes d'identité (2000-2001)
}

\author{
Isabelle Dépret
}

1 En Grèce, les dernières années du $\mathrm{XX}^{\mathrm{e}}$ siècle sont marquées par de vives tensions entre l'Église chrétienne orthodoxe - une Église étroitement liée à l'État - et le cabinet socialiste de Kostas Simitis. Le rapport de force est, à vrai dire, en place dès l'intronisation du nouvel archevêque d'Athènes, Mgr Christodoulos, en mai 1998. Néanmoins, peu après les législatives d'avril 2000, la situation se radicalise. Le conflit se cristallisera ici autour d'une annonce gouvernementale : la confession, une information jusqu'alors systématiquement précisée, ne figurera pas sur les prochaines cartes d'identité. Cette mesure, qui a pour contexte une Europe en construction, reflète tout d'abord un processus de standardisation normative dans un espace interétatique sans frontières. Le haut clergé orthodoxe de Grèce, qui dénonce un acte autoritaire, choisira cependant d'y résister : pendant plus d'un an, le Synode ${ }^{1}$ assumera ainsi un combat « inflexible ", au nom de l'hellénisme. Le litige n'en perd pas moins de son acuité dès l'été 2001. Le repli opéré, sur ce terrain, par les instances synodales fut volontiers assimilé à un constat d'échec ${ }^{2}$. Dans cet affrontement, une lente transformation des rapports entre État et religion en Grèce peut être perçue. L'intégration européenne, la sécularisation de la société, le thème, émergent, de la neutralité confessionnelle de l'État, participent de ces recompositions. L'issue de ces transformations, reste, soulignons-le, en partie indéterminée ${ }^{3}$.

2 Il est également possible d'envisager ce conflit comme l'expression d'enjeux internes à l'Église. Cette deuxième lecture, complémentaire, sera examinée ici. Un aspect, apparemment en marge de la "bataille», nous permettra d'aborder la question. L'attention portera sur le patriarche orthodoxe d'Istanbul/Constantinople. Ce dignitaire ecclésiastique se situe dans un double rapport, d'intériorité et d'extériorité, à l'Église orthodoxe de Grèce. Cette situation renvoie aux circonstances de la formation de l'État grec moderne, dans un espace jusqu'alors ottoman. 
Il nous faut, tout d'abord, revenir sur ce litige, entre pouvoir «temporel » et Église dominante, pour repérer ensuite une seconde ligne de clivage: plus latente, celle-ci traverse l'Église, où la figure du patriarche, Mgr Bartholomaios, tend à cristalliser un courant d'opposition à la direction officielle. Nous montrerons en quoi ces clivages réactivent une polarisation plus ancienne et témoignent d'enjeux institutionnels, dans l'Église orthodoxe de Grèce, au seuil du XXI siècle.

Le litige des cartes d'identité : la disparition de la donnée confessionnelle

4 Au printemps 2000, la Grèce, membre de la Communauté européenne depuis 1981, membre à part entière de l'espace Schengen depuis le mois de mars ${ }^{4}$, s'apprête à intégrer la zone euro. Le pays est gouverné par le Mouvement Socialiste Panhellénique (PASOK) et spécialement par son aile «moderniste», qui s'est imposée depuis janvier 1996. Des élections législatives sont prévues, le 9 avril. Elles sont remportées par le PASOK, qui obtient $43,79 \%$ des suffrages exprimés, contre $42,73 \%$ pour la formation conservatrice Nea Dimokratia ${ }^{5}$.

5 Peu après l'annonce des résultats, l'archevêque d'Athènes, puis le Saint-Synode, adressent de «chaleureuses félicitations » au Premier ministre, Kostas Simitis, reconduit dans ses fonctions ${ }^{6}$. Le 13 avril 2000, la prestation de serment devant le haut clergé orthodoxe consolide, conformément aux usages, l'entrée en fonction d'un nouveau cabinet. Dès le mois suivant, les relations entre les deux instances se détériorent pourtant de manière spectaculaire. Dans ce basculement, plusieurs facteurs joueront un rôle catalytique.

6 Le nouveau cabinet inclut un petit nombre de personnalités directement issues du milieu académique - et non parlementaire. C'est le cas du ministre de la Justice. Michalis Stathopoulos est, depuis 1976, professeur de droit public à l'université d'Athènes. Ses liens avec le $\mathrm{PASOK}^{7}$, son engagement en faveur des droits de l'homme participent de son personnage public, de même que ses convictions laïques. Celles-ci sont formulées, sans aucune ambiguïté, dans une étude juridique publiée en 1993. L'auteur y présente un projet de dissociation graduelle entre Église et État en Grèce ${ }^{8}$. Cette démarche réformatrice le place d'emblée dans un rapport revendicatif et en puissance conflictuel, à l'Église orthodoxe ${ }^{9}$. Le ministre de la Justice n'a-t-il pas, précisément, le profil de ces " intellectuels soi-disant modernistes", que raille volontiers l'archevêque d'Athènes, dans ses sermons en chaire ${ }^{10}$ ?

7 Début mai 2000, le climat s'alourdit. Dans un entretien accordé à un quotidien généraliste, le ministre de la Justice souligne la nécessité d'une réforme juridique et institutionnelle cohérente, dans le sens d'une sécularisation de l'État. Parmi les points évoqués ${ }^{11}$, le ministre précise que l'indication confessionnelle, qui apparait sur les cartes d'identité, ne s'inscrit pas dans la légalité. Cette mention violerait, en effet, de manière flagrante une loi adoptée en 1997 par le Parlement grec ${ }^{12}$.

8 La loi N.2472/1997, à laquelle se réfère M. Stathopoulos, enregistre l'essor des systèmes d'information électronique. Elle reconnaît la nécessité, pour toute personne, d'être protégée contre de possibles abus, liés à la circulation de données la concernant. Comme dans d'autres pays d'Europe, cette réflexion éthique et politique s'insère dans une problématique de garantie des droits individuels. Associée à la ratification des Accords de Schengen, la loi de décembre 1997 institue une Haute Autorité pour la Protection des Données Personnelles ${ }^{13}$. C'est précisément cette instance que saisit le ministre de la Justice, en mai 2000, à propos des nouvelles cartes d'identité ${ }^{14}$. 
Dès le 8 mai, la direction de l'Église orthodoxe souligne un décalage - suspect - entre les propos «offensifs» de M. Stathopoulos et les positions plus conciliantes formulées, jusqu'alors, par le PASOK. Cette formation ne se serait-elle pas engagée à maintenir intact, après avril 2000, le statu quo qui régit, en Grèce, les rapports Église et État ${ }^{15}$ ? Le métropolite de Thessaliotide, Mgr Theoklitos dénonce pour sa part une « attaque perfide contre l'Orthodoxie et l'Hellénisme ${ }^{16}$. La Constitution grecque de juin 1975 précise qu'en Grèce, la religion « dominante » est celle de l'Église orthodoxe orientale du Christ. Le droit public hellénique garantit, par ailleurs, à l'institution ecclésiastique une large autonomie, au sein de l'appareil d'État ${ }^{17}$.

Le 15 mai 2000, à l'issue d'une réunion attendue, la Haute Autorité pour la Protection des Données Personnelles déclare illégale la saisie, sur les cartes d'identité, de la confession; du nom du conjoint ; de la profession ; de l'empreinte digitale ; de l'adresse de résidence ; de la nationalité de l'individu. Cet avis, activation de la loi de 1997, engagerait l'État ${ }^{18}$. La sentence, qu'enregistre aussitôt le gouvernement, doit entrer en application dans les mois qui suivent ${ }^{19}$.

11 En Grèce, la délivrance de cartes d'identité spécifiant la confession individuelle remonterait au tournant des années 1930 et 1940. Le point d'origine de cette pratique circonstances et date exacte - n'est toutefois pas entièrement éclairci en 2000-2001 ${ }^{20}$. La fin des années 1960 consolide un cadre réglementaire qui, par la suite, ne sera que partiellement démantelé ${ }^{21}$. La confession individuelle n'apparaît pas, précisons-le, sur les passeports, confirmant le poids des enjeux internes, auxquels la variable religieuse s'est trouvée corrélée. Les premiers questionnements relatifs au maintien - ou non - de cette donnée sur des documents civils datent du milieu des années $1980{ }^{22}$. Depuis 1985-1986, les autorités séculières ont tenté à plus de dix reprises de modifier un dispositif en place depuis 1945. Jusqu'en mai 2000, cependant, toutes ces tentatives se sont avérées infructueuses, en raison de pressions contraires, pressions effectives ou pressions redoutées. Dans ce front d'opposition, il faut signaler le rôle, très ferme, de la hiérarchie ecclésiastique orthodoxe. Jusqu'à la fin des années 1990, celle-ci intervient régulièrement pour que la confession conserve, sur ces documents officiels, son statut d'information obligatoire.

Les cartes d'identité émises après 2000 ne préciseront donc plus l'appartenance religieuse du titulaire. Cette évolution, que certains n'envisageaient plus ${ }^{23}$, s'impose en quelques jours, sous forme de procédure administrative interne. Rendue superflue par cette double légitimité de la loi et de l'expertise juridique, une nouvelle discussion parlementaire est ainsi évitée ${ }^{24}$. L'Église n'est pas non plus consultée, dans un domaine où l'autorité séculière se pose, ainsi, comme seule compétente. Or, pour la direction ecclésiastique, une telle logique est inadmissible. Nous ne nous étendrons pas ici sur cette confrontation théâtralisée, sinon pour en relever deux aspects.

13 Il faut souligner, d'une part, l'importance de la parole publique, dimension du conflit et ressource, pour l'Église, dans la bataille. De mai 2000 à l'été 2001, l'argumentation du saint-synode est centrée sur un registre identitaire. La disparition, sur les cartes d'identité, de la donnée confessionnelle est présentée comme une soumission, totalement injustifiée, à des modèles "étrangers", comme une atteinte à "l'Orthodoxie et l'Hellénisme » ${ }^{25}$. La survie même de la Nation, touchée dans ses attributs identitaires les plus fondamentaux serait, à terme, menacée ${ }^{26}$. Au-delà, cette mesure est lue comme un indice et trahirait un projet cohérent. Ce point apparaît dans l'encyclique, émise le 6 juin 2000, par l'assemblée des métropolites, convoqués en séance plénière extraordinaire : 
La tactique intransigeante du gouvernement sur la question (...) vient conforter les soupçons de l'Église, selon lesquels la disparition de la confession sur les cartes d'identité constitue la première mesure d'une longue série, qui prévoit, en pratique, de pousser la religion aux marges de la vie publique, sociale et nationale avec toutes les conséquences que ceci implique pour notre Peuple. Cette attitude s'oppose aux convictions de notre Peuple fidèle, qui n'est pas disposé à se séparer, par la force, du sein vivifiant de la Mère Orthodoxie (...). Si, contrariant nos espoirs, une telle orientation était maintenue, il est évident que le Peuple perdrait un élément fondamental de son identité (...). En Grèce, le racisme, l'intolérance et la xénophobie n'ont jamais pu se développer. Nous, les Orthodoxes, avons toujours été les protecteurs des petits et des faibles (...). La Hiérarchie pense que dans ces heures critiques (...) cette mesure spécialement inopportune et irréfléchie a créé une affaire grave qui risque de diviser le Peuple et d'altérer la cohésion de notre tissu social ${ }^{27}$.

En 2000-2001, l'Église orthodoxe de Grèce plaide pour le maintien à titre optionnel (et non obligatoire) de cette information, sur les cartes d'identité ${ }^{28}$. Or, cette position constitue un infléchissement, aussi modeste soit-il, les autorités ecclésiastiques ayant jusqu'alors, toujours défendu le caractère impératif de cette information. Il faut relever le souci, pour l'Église, d'ancrer sa "lutte» dans le cadre de l'État de droit libéral, de la démocratie représentative. Il s'agit, au total, d'une argumentation principalement séculière, susceptible de toucher une fraction très large de la population, notamment les orthodoxes peu pratiquants, voire peu croyants.

Ces discours participent, ici, d'une stratégie de mobilisation du "peuple grec ", contre cette mesure gouvernementale. L'organisation de deux « rassemblements populaires » à Thessalonique puis à Athènes en juin 2000, le lancement, en septembre 2000, d'une vaste pétition, à l'échelle du pays, en attestent. Ces actions sont impulsées par les instances centrales de l'Église orthodoxe de Grèce ${ }^{29}$. Les signatures, encouragées à l'occasion des services liturgiques, sont d'abord recueillies dans le cadre paroissial, principalement dans les églises; les bulletins, rassemblés et contrôlés à l'échelle du diocèse, sont ensuite comptabilisés, traités par les services centraux de l'Église orthodoxe de Grèce. Les structures ecclésiastiques nationales, ramifiées sur l'ensemble du territoire, permettent cette opération. La réception des formulaires, prévue jusqu'au 25 mars 2001, sera légèrement prolongée. Cette pétition revendique la tenue d'un référendum national sur la question ${ }^{30}$.

Cette mobilisation ne suffira pas, pourtant, à infléchir les choix gouvernementaux. Au cours de l'année 2001, la hiérarchie orthodoxe essuiera, sur ce front, plusieurs revers décisifs. Le 21 mars 2001, tout d'abord, la thèse de la « mention facultative ", que prône le saint-synode, est récusée par le Conseil d'État ${ }^{31}$. Dans un avis, cette instance définit la religion comme une "donnée personnelle sensible qui relève de la conscience de l'individu face au divin ». Aussi, son inscription, même facultative, sur les cartes d'identité serait-elle anticonstitutionnelle. En effet, le dilemme auxquels se trouveraient confrontés les citoyens - inscrire ou non leur appartenance confessionnelle constituerait en soi une « contrainte de conscience » et entraînerait des « discriminations touchant ceux qui refusent de répondre ». La suppression, sur les papiers d'identité, de la nationalité est en revanche invalidée ${ }^{32}$.

Mesure strictement administrative pour le gouvernement, le thème s'est insinué dans le jeu partisan. Le 28 août 2001, la hiérarchie orthodoxe annonce le nombre de signatures réunies dans le cadre de la pétition : $3008901^{33}$. Ce chiffre, est-il observé, dépasse le nombre de voix obtenues, le 9 avril 2000, par le parti au pouvoir ${ }^{34}$. Non sans 
déchirements internes, la Nea Dimokratia tendra, avant le 28 août, à cautionner la résistance de l'Église orthodoxe, en pointant la faible légitimité du gouvernement ${ }^{35}$. Le 29 août 2001, l'archevêque d'Athènes est, par ailleurs, reçu par le Président de la République. L'appui du chef de l'État, un ancien cadre du parti Nea Dimokratia, semble recherché par Mgr Christodoulos, qui présente le résultat de la pétition. Or, cet appui n'est pas obtenu. Le jour même, le président d'honneur de la Nea Dimokratia envenime la controverse ${ }^{36}$. Pour Konstantin Mitsotakis, en effet, l'Église orthodoxe a remporté une victoire morale ${ }^{37}$. Fin 2001, l'apaisement du conflit paraît donc sanctionner, pour l'Église, l'échec de l'opération. Pourtant, à considérer l'enjeu - sous-jacent - de la laïcité, le bilan mérite d'être nuancé. Le statut de l'Église orthodoxe dans l'État n'est aucunement remis en cause au début du XXI ${ }^{\mathrm{e}}$ siècle.

En 2000-2001, l'archevêque d'Athènes et la direction de l'Église de Grèce tendent à exalter cette image d'une "union sacrée »: celle d'une Église héroïque, combattante, unie, soudée devant l'ennemi. Or, cette représentation n'a pas entièrement reflété la réalité. C'est ici que vient s'insérer la figure de Mgr Bartholomaios, Patriarche de Constantinople depuis l'automne 1991.

L'insertion du patriarche de Constantinople dans le conflit

20 Au cours de cette crise, en effet, la posture du Patriarche orthodoxe d'Istanbul ne fut pas perçue, en Grèce, comme strictement neutre. Certes, c'est tout d'abord un appel à l'unité et à la concorde qui est lancé à l'adresse du peuple grec. Cet appel est réitéré, au fil de la période ${ }^{38}$. Pour autant, dès le mois de mai 2000, au moment où explose le conflit, Mgr Bartholomaios tend à se démarquer du combat engagé, à Athènes, par le Saint-Synode.

21 À l'occasion d'un sermon prononcé, le 28 mai 2000, à l'Institut théologique de Halki, près d'Istanbul, Mgr Bartholomaios, souligne que «l'Église orthodoxe ne désobéit pas aux lois des États dans lesquels elle se trouve ». Au cours de cette allocution, adressée, entre autres, " aux dirigeants et responsables de ce monde ", il reprend la formule attribuée à Jésus-Christ : "Rendez à César ce qui appartient à César et à Dieu ce qui appartient à Dieu ». L'apôtre saint Paul est également cité, justifiant une nécessaire loyauté au pouvoir séculier, pouvoir établi par Dieu. L'histoire multiséculaire de l'Église orthodoxe, ne témoignerait-elle pas, au demeurant, d'une attitude « respectueuse des lois séculières »? Ainsi, le précepte "Obéissez davantage à Dieu qu'aux hommes " ${ }^{39}$ ne s'appliquerait, selon Mgr Bartholomaios, qu'en cas de négation, par l'État, de la valeur du Christ. Pour les chrétiens, respecter les lois de leur pays, ne signifierait pas pour autant renoncer à leurs droits ${ }^{40}$.

Dans ces propos, transparaissent, tout d'abord, les préoccupations très directes $d u$ Patriarche : ce sont celles d'un citoyen turc, responsable d'une communauté religieuse largement minoritaire et sous surveillance. Depuis le début du XXe siècle, la communauté orthodoxe de Turquie s'est fortement réduite. Estimée, en 1927, à plus 109905 fidèles, dont 100214 à Istanbul, elle ne compte que quelques milliers de personnes, dans les années $1990^{41}$. Au XXe siècle, le Patriarcat, en raison même de sa position minoritaire, de ses liens possibles avec des puissances ou des réseaux « étrangers ", a plutôt été considéré avec méfiance par l'État turc ${ }^{42}$. C'est donc en premier lieu dans le cadre de la Turquie que Mgr Bartholomaios inscrit ses fonctions ecclésiastiques et défend les droits des chrétiens orthodoxes ${ }^{43}$.

23 Toutefois, perçus de Grèce, les propos de Mgr Bartholomaios sonnent plutôt comme un rappel à l'ordre subtil. En justifiant une séparation rigoureuse entre les compétences du 
pouvoir temporel et celles du pouvoir spirituel, en rappelant que l'Église doit se soumettre aux lois de l'État, le Patriarche semble ici désavouer la direction de l'Église de Grèce. Cette lecture tend à s'affermir de mai 2000 à août 2001 et ses implications méritent d'être envisagées. Nous attirons l'attention sur trois aspects.

En premier lieu, le hiatus, perçu entre le Synode de l'Église orthodoxe de Grèce et le Patriarcat a fourni au gouvernement un levier dans ce bras de fer. En second lieu, la figure du Patriarche « œcuménique » tendra, une fois de plus, à cristalliser en Grèce une opposition à l'archevêché orthodoxe d'Athènes. Il faut aussi se demander dans quelle mesure cette polarisation n'a pas, par là même, contenu les dissensions au sein - et non à l'extérieur - de l'Église. En ce sens, contester les orientations de l'Église « helladique » ne signifie pas, en Grèce, rejeter l'Église orthodoxe.

Au printemps 1987, l'Église Orthodoxe de Grèce se trouvait engagée dans une autre bataille face au gouvernement. Quelques mois plus tôt, en effet, le cabinet socialiste Andreas Papandreou présentait, sur un dossier sensible, un projet de loi. Intitulé "Règlement de questions touchant à la fortune monastique et ecclésiastique », le projet, introduit par le ministre de l'Éducation nationale et des Cultes Antonis Tritsis, proposait une évaluation, concertée mais systématique, du patrimoine foncier de l'Église orthodoxe, en Grèce, dans le but avoué d'en améliorer la gestion. Était ici envisagée, entre autres, la cession par l'Église des $4 / 5^{\mathrm{e}}$ de la fortune forestière et monastique, soit environ 150000 hectares de terres. Ce transfert était prévu, sous réserve de compensation, en échange de l'acquisition formelle, par l'Église, des droits de propriété sur les surfaces restantes ${ }^{44}$. De facto, la hiérarchie orthodoxe de Grèce refusait vigoureusement ce texte. Aspect d'une stratégie de résistance multiforme ${ }^{45}$, le Patriarcat d'Istanbul et les autres Églises orthodoxes étaient, là aussi, alertés et appelés en renfort. Point intéressant, dénonçant une "tentative de capture " perpétrée par l'État, l'Église de Grèce menaçait alors de renoncer à son statut d'Église autocéphale. Elle se serait replacée sous l'autorité spirituelle directe du Patriarche de Constantinople. Ce changement de statut lui aurait permis de se soustraire, opportunément, aux implications de la loi Tritsis : les territoires, tels le Mont Athos, sous juridiction ecclésiastique patriarcale, étaient, en effet, exclus des mesures stipulées ${ }^{46}$. Au printemps 1987, le patriarche de Constantinople, Mgr Dimitrios, avait clairement apporté son appui à l'Église de Grèce ${ }^{47}$. La situation du gouvernement s'en était trouvée vulnérabilisée face aux électeurs orthodoxes, face à l'opposition politique interne ${ }^{48}$.

En 2000, la configuration semble bien différente : la bataille, lancée autour des cartes d'identité, ne semble pas cautionnée par la «Grande Église de Constantinople ». La validité du combat se voit contestée sur le terrain ecclésiastique orthodoxe lui-même. Cette prise de position, qui vient souligner des divisions internes ne peut, en première lecture, qu'affaiblir l'Église orthodoxe de Grèce dans son procès. Elle offre, simultanément, aux autorités séculières des marges de manœuvre. Ainsi, le bras de fer qui a opposé le gouvernement et la hiérarchie de l'Église dominante, n'a pu être assimilé à un combat - beaucoup plus dangereux - entre État et religion chrétienne orthodoxe.

La figure du Patriarche, un autre point de ralliement

D'une manière générale, une attitude réprobatrice à l'égard du saint-synode de l'Église de Grèce - ou de l'archevêché d'Athènes - a pu s'exprimer, à travers la figure du Patriarche de Constantinople. Ce dernier est ici valorisé comme personnalité ou comme symbole d'une institution. Le phénomène concerne donc, durant cette période, les milieux laïques. Il apparaît dans la presse généraliste et peut être observé parmi les Grecs de culture 
orthodoxe, y compris dans des milieux peu pratiquants, sinon peu croyants. La tendance mérite, à ce titre, d'être signalée. Ainsi, à l'image de l'archevêque d'Athènes, dénoncé comme " nationaliste ", « conservateur ", « arrogant ", « ambitieux », « intransigeant » tend à être opposée la figure du Patriarche Bartholomaios. Ce dernier, dont, par contraste, les positions foncièrement opposées au nationalisme, "progressistes", «tolérantes », " ouvertes ", « universalistes/œcuméniques » sont soulignées, se présente, ici, comme un contre modèle, face à Mgr Christodoulos. D'autres formes de contestation se sont manifestées, à cette occasion. Afficher, notamment, une posture d'indifférence, de désintérêt ou de distance dépréciative a pu constituer un mode de contestation : l'affaire est alors renvoyée à des enjeux " politiques » c'est-à-dire, à des enjeux de pouvoir ou à des conflits de personnes. Elle n'aurait, par conséquent, rien à voir avec l'orthodoxie et n'aurait que peu d'intérêt. Les parties adverses du litige sont alors renvoyées dos à dos et placées, négativement, sur le même plan.

Observable dans l'espace laïque, cette polarisation conflictuelle entre archevêché d'Athènes et Patriarcat de Constantinople est également perceptible, mais beaucoup plus subtilement, dans le clergé et notamment, le haut clergé orthodoxe de Grèce. La mise en avant d'une affiliation privilégiée à la "Grande Église de Constantinople » - attitude, au demeurant, parfaitement orthodoxe - se serait chargée ici d'un sens particulier: elle aurait, là aussi, permis de marquer une distance. Ces réserves semblent porter sur deux aspects majeurs: d'une part, les méthodes, sinon les positions du Saint-Synode Permanent durant cette période; d'autre part, le pouvoir, jugé abusif, de l'archevêché $\mathrm{d}$ 'Athènes, que contestent certains évêques.

Durant la période qui nous intéresse, il convient d'emblée de relativiser les cas de dissidence dans la hiérarchie orthodoxe de Grèce : les expressions d'opposition ouverte se sont avérées, au total, très peu nombreuses. Pour autant, dès le printemps 2000 , quelques voix discordantes se font entendre. Un petit nombre de métropolites exprime des réticences, quant au style, aux méthodes, voire aux choix de la direction ecclésiastique. Cette brèche apparaît en juin 2000. Quatre métropolites, à contre-courant des instructions officielles, annoncent qu'ils n'exhorteront pas, quant à eux, les fidèles à s'associer aux rassemblements de Salonique et d'Athènes: ce sont les métropolites de Iannina (Mgr Theoklitos), de Thèbes (Mgr Ieronymos), de Zakynthos (Mgr Chrysostomos) et de Preveza (Mgr Meletios). Le 12 juin 2000, devant les media, le métropolite de Iannina justifie sa position. Selon lui, «le contenu des cartes d'identité relève des affaires de l'État ». Plus généralement, «l'Église ne devrait pas s'engager en politique (...) Alors que l'Église s'efforce d'entrer en confrontation avec le pouvoir séculier, ce dernier (...) ne se présente pas comme un rival mais comme une institution responsable du destin du peuple ${ }^{49}$.

30 Quelques jours après la grande manifestation d'Athènes, à la fin juin 2000, les métropolites de Iannina, de Thèbes et de Zakynthos reprochent à Mgr Christodoulos d'avoir brandi, devant la foule, l'étendard de la Grande Laure ${ }^{50}$. Ce geste rappelle, certes, une image symboliquement forte, ancrée dans la légende nationale : celle de l'évêque de Patras Mgr Germanos levant, selon la tradition, le 25 mars 1821, l'étendard de la révolte contre «l'oppresseur turc " ${ }^{51}$. Parce qu'elle puise dans un registre symbolique, affectif, dans une mémoire périodiquement réactivée, l'image peut être efficace et mobilisatrice. Le parallèle est-il néanmoins opportun? Ne présente-t-il pas, précisément, une Église soulevant le peuple contre l'autorité séculière; un haut clergé ethnarque, porteur d'un mouvement politique, voire d'un mouvement guerrier? Les trois métropolites 
reprochent, par ailleurs, à Mgr Christodoulos son absence remarquée, quelques jours plus tôt, lors de la cérémonie d'entrée de la Grèce dans l'Union Monétaire Européenne ${ }^{52}$. Les réserves exprimées par ces métropolites concerneront, de même, la campagne de collecte de signatures décidée par le Synode Permanent en été 2000. Or, les positions du patriarche Bartholomaios s'ajustent bien ici à la posture dissidente qu'adoptent ces trois évêques.

31 Le passage de Mgr Bartholomaios à Athènes en octobre 2000 permet d'observer cet écheveau de relations - ou de stratégies. Il s'insère dans le cadre d'une visite de huit jours envisagée, semble-t-il, avec réticence par l'Église de Grèce ${ }^{53}$. Le 18 octobre 2000, veille de l'arrivée de Mgr Bartholomaios, quelques personnalités politiques de gauche, militants, intellectuels, réunis à l'initiative de la députée européenne Anna Karamanou (PASOK) se déclarent prêts à fonder un "Front de la Raison ». L'un des principaux intervenants, Léonidas Kyrkos, parle d'une «exacerbation du fondamentalisme chrétien, sous la direction de Christodoulos », qui constituerait « l'un des principaux dangers pour la Grèce d'aujourd'hui » ${ }^{54}$.

Le caractère privé de cette visite, pourtant souligné, doit être relativisé. Mgr Bartholomaios est accueilli à l'aéroport par l'archevêque d'Athènes, ainsi que par une délégation ministérielle, conduite par le ministre des Affaires étrangères, Georges Papandreou. Au cours de ce séjour, Mgr Bartholomaios rencontre le Président de la République, le Premier ministre, le ministre des Affaires étrangères, le Président du Parlement, les dirigeants de plusieurs partis politiques, le maire d'Athènes. Ces entretiens comportent un contenu politique et diplomatique explicite : les relations gréco-turques, la situation dans les Balkans, celle des Grecs dans le monde figurent parmi les questions discutées. Invité à un repas offert par le Premier ministre en son honneur, Mgr Bartholomaios observe qu'au « XXI ${ }^{\text {e }}$ siècle, les peuples ne souhaitent pas que l'Église Orthodoxe soit simplement une autre puissance temporelle et une machine de combat». Il précise que "naturellement, le Phanar ne se mêle pas de politique ${ }^{55}$. Plusieurs responsables politiques reviennent pourtant sur ces propos. C'est le cas du Premier ministre, du président du Parlement (Apostolos Kaklamanis) ou du président de la Coalition de la Gauche et du Progrès (Nikos Konstantopoulos), qui déclarent s'y rallier pleinement ${ }^{56}$. La médiation du Patriarche œcuménique aurait-elle facilité une rencontre et, peut-être, un rapprochement entre l'archevêque d'Athènes et Kostas Simitis? Ce ne fut, semble-t-il, pas vraiment le cas. Ce séjour marque aussi l'inauguration d'un Bureau du Patriarcat d'Istanbul à Athènes.

33 La petite ville de Sappès, située en Thrace dans le département du Rhodope, constitue une étape dans la visite de Mgr Bartholomaios en Grèce. Le maire de ce village, Dinos Charitopoulos, justifie sa fidélité primordiale au Patriarche, en avançant deux motifs, qui étayent notre propos. D'une part, en effet, la commune s'inscrit en « nouvelle Grèce » et les diocèses correspondants relèvent toujours «du Patriarcat». D'autre part, "les convictions exprimées par le Patriarche (...) correspondent mieux aux sociétés de droit et au monde contemporain (...) ». Le maire de Sappès ajoute : "Nous sommes prêts à nous replacer, y compris d'un point de vue administratif, sous l'autorité de l'église de Constantinople ${ }^{57}$. Mgr Bartholomaios, pour sa part, valorise dans cette commune un «modèle de société multireligieuse et multiculturelle». Il souligne le développement d'un réseau de coopération et d'échange entre municipalités de Thrace occidentale (en Grèce) et de Thrace orientale (en Turquie), projet dans lequel le maire de Sappès s'est engagé ${ }^{58}$. Dans cet exemple, l'affirmation d'un attachement primordial au patriarcat 
s'articule à une critique, sous-jacente, du Synode de l'Église de Grèce et de ses orientations: et ceci a fortiori parce que nous sommes ici en Thrace, petite patrie de l'archevêque d'Athènes.

Fin avril 1998, le métropolite Christodoulos Paraskevaïdis était promu par ses pairs au siège archiépiscopal d'Athènes. Il s'agit d'une position clé dans cette institution. On peut se demander dans quelle mesure le défi lancé, deux ans plus tard, aux autorités politiques ne s'inscrit pas dans un projet sous-jacent, de rassemblement. Une mise en perspective historique nous permettra d'étayer l'argument. Nous rappellerons, tout d'abord que l'Église autocéphale de Grèce s'établit au début du XIX siècle, en se soustrayant de l'autorité du Patriarcat de Constantinople. Nous préciserons, ensuite, que cette émancipation (ambiguë) ne fut jamais complète. Nous soulignerons, enfin, qu'au terme du $\mathrm{XX}^{\mathrm{e}}$ siècle, les transformations de l'espace européen remettent en cause certains équilibres, au sein de l'Église orthodoxe.

Retour sur une autonomisation fondatrice

L'Église orthodoxe de Grèce émerge au début du XIXe siècle, dans le contexte d'une désagrégation de l'empire ottoman ${ }^{59}$. Trois processus marquants accompagnent la création d'un État grec, dont le principe de légitimation est national : d'une part, une logique d'indépendance ecclésiastique, conçue comme aspect de l'indépendance politique ; d'autre part, une reprise en main, par les autorités séculières, d'une partie des fonctions jusqu'alors assumées par le clergé. En corollaire, un statut d'Église officielle est reconnu à l'Église orthodoxe. Ces processus se sont imposés sur fond d'importantes dissensions, au sein du millet chrétien orthodoxe. Détachée au plan spirituel de l'Église de Constantinople, alors associée au pouvoir ottoman, cette instance est placée sous le contrôle du souverain temporel ${ }^{60}$. Le synode de l'Église de Grèce n'est reconnu par le Patriarcat qu'au début des années 1850.

L'Église orthodoxe de Grèce peut être envisagée sous le prisme d'un conflit initial entre deux sources d'autorité : un pouvoir récent, les autorités civiles du nouvel État grec, et un pouvoir traditionnel, le Patriarche de Constantinople. D'une manière ou d'une autre, ces deux autorités peuvent alors prétendre représenter - ou diriger - la communauté nationale hellénique, une communauté qui se redéfinit alors. Aux premiers temps de l'État, l'autorité, d'une part, du roi de Grèce, d'autre part du Patriarche, tendent à se présenter, pour le clergé grec orthodoxe, comme deux allégeances opposées, mutuellement exclusives. L'adhésion du clergé orthodoxe à l'État grec moderne constitue tout sauf une évidence, en première moitié du XIX siècle. Aspect du litige certes, l'origine " étrangère » des premiers souverains de Grèce ne suffit pas à rendre compte de ces rapports conflictuels. Dès le début du XIXe siècle, néanmoins, plusieurs conceptions nationales grecques s'affirment et certaines d'entre elles valorisent la tradition religieuse. Dans ce contexte, l'Église orthodoxe constituera le lieu crucial - symbolique d'une tension entre une logique d'État-nation et une logique impériale ${ }^{61}$.

Les contours de la Grèce ne se stabilisent qu'après la Seconde Guerre mondiale. Outre l'assurance d'une protection institutionnelle renforcée, un autre facteur a favorisé l'insertion du clergé orthodoxe dans l'État et dans ses projets. Le principe d'une coïncidence entre territoire politique et territoire ecclésiastique - qui avait justifié l'autocéphalie - a impliqué, de même, l'ajustement quasi automatique des frontières de l'Église dominante sur celles de l'État. Ainsi, en 1866, deux ans après le rattachement des îles ioniennes à la Grèce, les diocèses correspondants sont cédés à l'Église de Grèce par un Tomos du Patriarche Sophronios III. En 1882, un an après l'annexion de la Thessalie et 
d'une partie de l'Épire, les diocèses correspondants, jusqu'alors sous juridiction du Patriarcat de Constantinople, sont rattachés à l'Église « d'Athènes ».

Comme structure territorialisée, instance de pouvoir et représentante d'une communauté de fidèles, l'Église autocéphale orthodoxe de Grèce se développe bien dans l'ombre de l'État, au XIX et au XXe siècle. Jusqu'aux premières décennies du XXe siècle, les frontières de la Grèce sont en expansion.

Pour autant, le cordon ne fut jamais rompu avec le Patriarcat. Après 1912, la logique d'alignement du territoire ecclésiastique sur celui, administratif, de l'État se brouille. Les régions acquises après les guerres balkaniques de 1912-1913, sont restées formellement des métropoles du Patriarcat de Constantinople. Cette configuration renvoie au contexte social, religieux, politique des années 1920 et 1930. Elle éclaire un système ecclésiastique éclaté, toujours en vigueur aujourd'hui.

Jusqu'au début des années 1920, l'annexion des côtes micrasiatiques semble en effet possible ${ }^{62}$. Cette perspective s'effondre en 1922 . Lancée dès 1919 , la guerre hellénoturque débouche, en août et septembre 1922, sur une débâcle pour les troupes grecques ${ }^{63}$. Dans les années 1920-1930, ébranlé par ce qui sera qualifié de "catastrophe nationale ", l'État hellénique se trouve au défi d'intégrer - économiquement, socialement, culturellement, politiquement - un million et demi de réfugiés, appauvris et déracinés. Ces immigrés viennent principalement d'Asie mineure - plus de 1,1 million - mais aussi de Bulgarie, de Russie, d'Arménie. Une majorité s'installera, précisément, dans les régions fraîchement acquises : la Thrace occidentale, la Macédoine méridionale, les îles de l'Égée, une partie de l'Épire ${ }^{64}$. Il s'agit alors d'espaces multicommunautaires, où l'autorité d'Athènes est encore fragile. Par ailleurs, en 1923, le Patriarche orthodoxe de Constantinople perd son statut d'ethnarque, pour devenir un (simple) chef religieux, dans la nouvelle République de Turquie ${ }^{65}$.

41 L'Acte synodal et patriarcal du 4 septembre 1928 est émis dans ce contexte. Ce texte concerne les régions incorporées à la Grèce après 1912 et précise leur régime ecclésiastique. Par ce document, que négocient l'État grec et le Patriarcat, ce dernier confie, par mandat, à titre provisoire et sous conditions, l'administration des « Nouvelles Provinces » à l'Église autocéphale de Grèce. Ces conditions visent à préserver l'autorité spirituelle patriarcale, fut-elle, désormais, formelle. Parmi ces conditions, est notamment posée l'égale représentation de ces provinces dans les instances administratives centrales de l'Église orthodoxe de Grèce. Cette parité se veut non seulement quantitative - un nombre égal de métropolites - mais aussi qualitative. L'affectation à des postes clés est ici visée. Par ailleurs, lors des services liturgiques, c'est le nom du Patriarche - et non celui du métropolite d'Athènes - qui est, en premier lieu, invoqué. Mérite également mention la possibilité, offerte aux prêtres des " Nouvelles Provinces ", de saisir en appel la justice ecclésiastique du Patriarcat; ceci, pour contester une décision judiciaire de l'église orthodoxe de Grèce.

42 Au cours de cette période, une autre tendance est perceptible : il s'agit d'un renforcement des positions de l'évêque d'Athènes. La Charte ecclésiastique adoptée en 1923 en atteste : face aux autres évêques de l'Église orthodoxe de Grèce, se distingue celui de la capitale élevé, à cette date, au rang d'Archevêque. Or, à la fin du $\mathrm{XX}^{\mathrm{e}}$ siècle, ces équilibres vacillent, alors même que l'espace européen se recompose. Au-delà du rapport aux fidèles, dont le profil, les attentes évoluent, se nouent aussi des enjeux d'autorité, sur une structure cléricale, sur des communautés et sur des territoires. 
L'Église orthodoxe et la recomposition de l'espace européen à la fin du xx siècle Le Patriarcat rappelle que les textes régissant le statut de l'Église de Grèce n'autorisent
pas l'évêque d'Athènes à porter le titre de "Premier ». Seule, par conséquent, une nouvelle Convention - ce qui suppose l'aval du Patriarcat - rendrait possible cette évolution. Or pour certains clercs, un tel amendement ne renforce pas seulement la position archiépiscopale d'Athènes : elle ouvrirait aussi à l'Église de Grèce la perspective de s'ériger, à moyen terme, en Patriarcat autonome. Mgr Christodoulos est alors invité à "éviter les nouveaux titres, non hérités de la tradition », qui pourraient déclencher "l'hostilité des fidèles » ${ }^{75}$. Dans les années 1998-2001, Mgr Bartholomaios rappelle, à 
plusieurs reprises, que « les Grecs n'ont qu'un seul Patriarche » ${ }^{76}$. Le Patriarcat - en tant qu'administration ${ }^{77}$ - semble soucieux de défendre des compétences prévues dans l'Acte de 1928 : un droit de contrôle sur les nominations d'évêques des "Nouvelles Provinces ", sur la composition du Synode Permanent de l'Église orthodoxe de Grèce; un droit d'information privilégié et de juridiction en appel dans les métropoles du «Patriarcat œcuménique ».

Ces rivalités latentes doivent être replacées dans le cadre, plus large, de l'Europe. Au cours du XIX ${ }^{e}$ siècle, l'Église de Constantinople, concurrencée depuis plusieurs siècles par le Patriarcat de Moscou, s'étiole ${ }^{78}$. Elle survit, de justesse, au démantèlement de l'empire ottoman. Inversement, pour l'Église de Grèce, les XIX et $\mathrm{XX}^{\mathrm{e}}$ siècles marquent une période de consolidation institutionnelle, administrative, spatiale. Au XIX siècle, les notions de "conservatisme ", de "réaction", "d'ancien régime", de "déclin » se trouvent plus volontiers associées au Patriarcat qu'à l'Église orthodoxe de Grèce. Comparée au Patriarcat, cette dernière instance semble davantage ajustée aux formes et aux valeurs d'une certaine modernité, aux tendances d'avenir. Deux siècles plus tard, ne serait-ce qu'en terme de représentations, la situation s'est profondément modifiée.

À la fin du XXe siècle, les transformations - géopolitiques, institutionnelles, normatives en cours, à l'échelle du continent européen tendent, au contraire, à réévaluer la figure $\mathrm{du}$ Patriarche orthodoxe d'Istanbul. L'évêque de Constantinople, symbole d'un passé prestigieux et, pour certains, point de ralliement pour une Église orthodoxe réunifiée ${ }^{79}$, est une autorité religieuse transnationale. Certains le définissent comme un "souverain déterritorialisé » ${ }^{80}$. Le Patriarche "œcuménique » serait-il mieux armé, dans cet environnement en gestation?

D'une part, en effet, en Europe orientale et balkanique, une certaine réaffirmation des sentiments et des pratiques religieuses a été observée, après la chute des régimes communistes. Depuis le début des années 1990, les Églises locales se reconstruisent. Or, le christianisme orthodoxe est, dans cette région, une composante culturelle. D'autre part, dans le sillage d'une Europe politique en formation, les frontières étatiques tendent à s'émousser : suppression des barrières entre pays, redistribution des compétences entre États et instances communautaires, rôle croissant des structures confédérales européennes. Ces processus, inscrits sur la longue durée, encouragent migrations, circulation, échanges, au sein de l'Union. Enfin, le nationalisme, associé, dans les années 1990, aux conflits balkaniques, tend à se charger d'une forte connotation négative. En tant que discours politique, il est volontiers stigmatisé, rejeté du côté de la fermeture, de l'intolérance, de la violence, de l'autoritarisme et du conservatisme politique. Face aux nationalismes, face aux frontières closes de la petite patrie ou de l'État nation "parfaitement homogène ", les idées supranationales se trouvent réévaluées, incarnant une forme de progrès, une ouverture constructive. Or, à la fin du $\mathrm{XX}^{\mathrm{e}}$ siècle, c'est le Patriarche de Constantinople, beaucoup plus que l'Église orthodoxe de Grèce, qui semble incarner ces valeurs et qui s'en réclame le plus explicitement.

51 Pour les autorités turques, l'autorité spirituelle de l'évêque orthodoxe d'Istanbul est locale. Elle s'étend sur la communauté grecque orthodoxe du diocèse d'Istanbul et elle seule, soit quelques milliers de personnes au plus ${ }^{81}$. Le Patriarche revendique pourtant une position honorifique primordiale dans le monde orthodoxe, justifiée par l'ancienneté, par l'histoire. Son espace juridictionnel serait beaucoup plus étendu. L'adjectif "œcuménique", auquel le Patriarcat est extrêmement attaché, reflète ces préoccupations. Primat d'honneur de plus de deux cents millions d'orthodoxes dans le 
monde, il possède, en ce sens, une stature universelle. Ceci vaut spécialement en Europe, où une partie des fidèles et du clergé (grecs) orthodoxes relève, en dernière instance, de son autorité spirituelle ${ }^{82}$. Dans les années 1990, le Patriarche orthodoxe de Constantinople, citoyen turc de culture grecque et orthodoxe, est, sans conteste, un chef religieux européen ${ }^{83}$.

L'Église orthodoxe de Grèce : Église autocéphale ou Église bicéphale?

La question de la présence de l'orthodoxie en Europe - présence spirituelle, culturelle, mais aussi institutionnelle et ecclésiastique - ne constitue pas seulement un facteur de solidarité entre les différentes Églises orthodoxes d'Europe. Elle met également à jour des divergences, parfois des rivalités, entre des Églises, qui cherchent leur place dans un ensemble politique en redéfinition. Face aux dogmes, qui soulignent l'unité fondamentale de l'Église, dans sa diversité et ses ramifications locales, se dressent des structures ecclésiastiques étatiques ou nationales, fort attachées à leur existence institutionnelle, à leurs spécificités : des instances peu disposées à perdre leur statut ou leur autonomie décisionnelle. Or, ces possibles enjeux d'autorité éclairent les rapports entre l'archevêque d'Athènes et le Patriarche de Constantinople. Précisément, leur proximité constitue ici une source potentielle de tension. L'affrontement qui se cristallise, en 2000-2001, autour des cartes d'identité, s'inscrit dans ce contexte. Le défi lancé par l'Église aux responsables civils grecs semble avoir sous-tendu un projet, à la fois personnel et institutionnel: affirmer l'autorité de l'archevêché d'Athènes et ce, en premier lieu dans l'Église orthodoxe.

\section{NOTES}

1. Sauf indication contraire, l'expression « Saint-Synode » désignera, dans cet article, le synode des métropolites de l'Église orthodoxe de Grèce.

2. Konstantinos Angelopoulos,

3. Isabelle Dépret, « Identité contre laïcité ? L'Église orthodoxe de Grèce et la "sauvegarde de l'identité nationale" à la fin du $\mathrm{xx}^{\mathrm{e}}$ siècle », Publications de l'Université C. Alfieri de Florence, Faculté de Sciences Politiques, Reprint Series n 37, 2005. Pour un examen plus approfondi, Isabelle Dépret, « Le Saint-Synode de l'Église orthodoxe de Grèce et les gouvernements socialistes 1998-2001. Appartenance religieuse, identité nationale, identité civile ", thèse de doctorat en histoire, Institut d'Études Politiques de ParisUniversité d'Athènes, Paris, mars-juin 2004. La réflexion porte, dans ce travail, sur le rôle ambigu de l'Église orthodoxe dans la construction d'un État et d'une communauté nationale, depuis le début du xix ${ }^{\mathrm{e}}$ siècle.

4. Les accords de Schengen entrent en vigueur en Grèce à partir du $1^{\mathrm{er}}$ janvier 1998.

5. ANA, 10-11 avril 2000. "Les élections du 9 avril 2000 », Ambassade de France à Athènes, Service de Presse.

6. Bureau de presse du Saint-Synode de l'Église de Grèce, 10 avril 2000. 
7. En 1985, il est, notamment, Secrétaire d'État chargé des relations avec la presse dans le gouvernement Andreas Papandreou. Michalis Stahopoulos est, par ailleurs, recteur de l'Université d'Athènes, de 1983 à 1991.

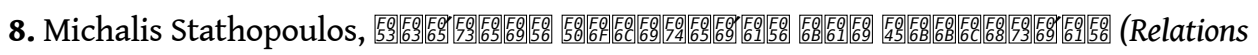
entre État et Église), Athènes, Komotini, 1993, 62 p.

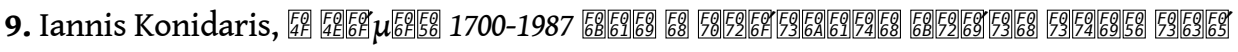

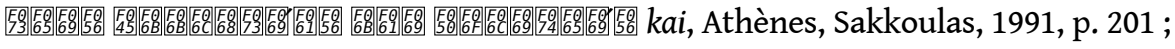

228-231.

10. Mgr Christodoulos, homélie dominicale du 14 mai 2000, Athènes.

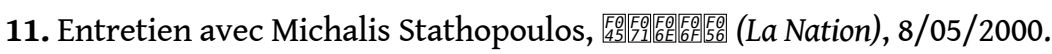

12. Le ministre se prononce aussi, à cette occasion, pour la légalisation en Grèce de funérailles civiles, la mesure ne fut-elle sollicitée que par quelques-uns. Il se déclare favorable à l'institution d'un serment civil, qui offrirait une alternative au seul serment religieux. Il exprime, quelques jours plus tard, son souhait d'impulser une révision du Code pénal hellénique. Les articles 198 et 199 du Code pénal qui sanctionnent « l'attentat contre la paix religieuse » sont spécialement visés ici.

13. Loi N.2472/1997 relative à la « protection de l'individu, lors de la mise en place de données à caractère personnel ».

14. Au printemps 2000 , cette instance, déclarée indépendante et composée de cinq membres, est présidée par Konstantin Dafermos.

15. Communiqué de la Hiérarchie orthodoxe à la presse du 8 mai 2000.

16. Net, 9/05/2000.

17. Notamment, «Constitution hellénique du 11 juin 1975 », article 3. Loi N.590/1977.

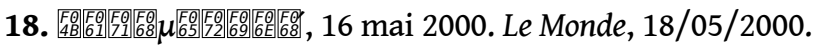

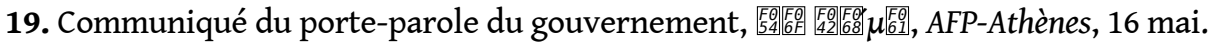
20. La loi 87/1945, qui impose le port d'une pièce d'identité, inclut la confession parmi les

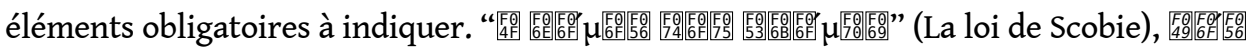

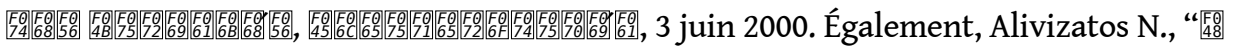

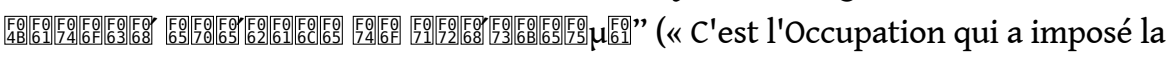

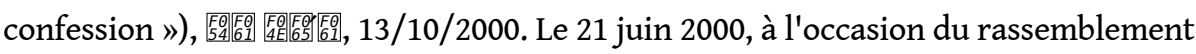
d'Athènes, Mgr Christodoulos affirme que la confession figure « depuis 80 ans » sur les bulletins d'identité civils.

21. Décret-loi 127/1969.

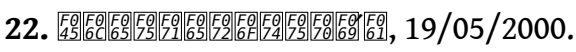

23. Didier Kunz, Le Monde, 17/05/2000.

24. Cette procédure se trouve contestée à ce titre. En début juin, l'homme politique de gauche Manolis Drettakis (Synaspismos) dénonce une mise à l'écart du Parlement dans la gestion du dossier. L'auteur reconnaît néanmoins qu'un projet de loi, qui proposerait de supprimer la mention confessionnelle, serait probablement rejeté par les députés.

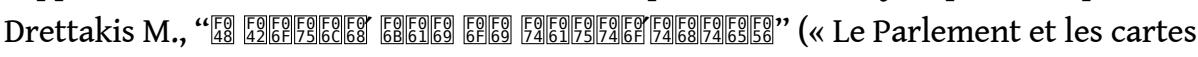

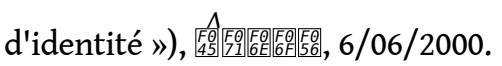

25. Net, 9/05/2000.

26. Secrétariat général du Saint-Synode, Athènes, 26/05/2000. 2000, p. 487-488.

27. Compte rendu de la séance extraordinaire du Saint-Synode de la Hiérarchie (ISI) de

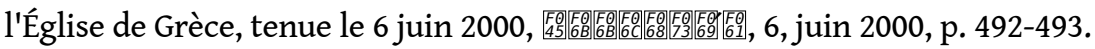

28. Ibid. 
29. Mgr Christodoulos, homélie prononcée le 14 septembre 2000, au monastère de

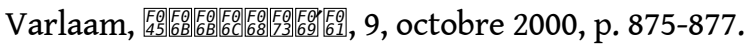

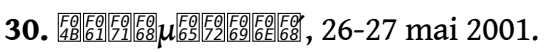

31. Cette instance, dont les jugements sont contraignants, statuait ici sur huit recours déposés, en décembre 2000, par des associations et des fidèles chrétiens orthodoxes.

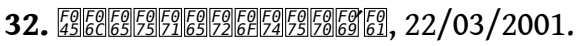

33. Déclaration de Mgr Christodoulos, Bureau de presse de l'archevêché d'Athènes, 28 août 2000.

34. Déclaration du porte-parole du Saint-Synode, « Station radiophonique de l'Église de

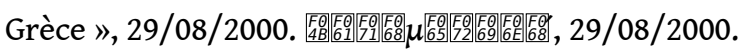

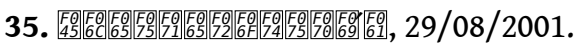

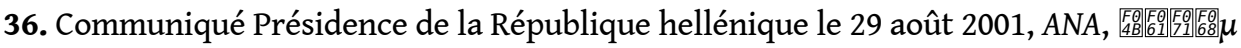

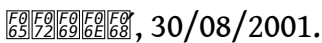

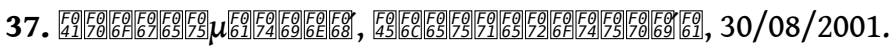

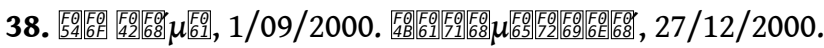

39. Nouveau Testament, Actes des Apôtres, 5-29.

40. Athens News Agency, 29 et 30/05/2000.

41. Samim Akgönul qualifie cette communauté de « minorité » en voie " d'extinction ». Samim Akgönul, « Les Grecs de Turquie. Processus d'extinction d'une minorité de l'âge de l'État-nation à l'âge de la mondialisation 1923-2001 », Thèse de Doctorat, Université Marc Bloch, Strasbourg, 2001.

42. Notamment, Harry PsOMIADIS, « The Ecumenical Patriarcate Under the Turkish Republic. The First Ten Years », Balkans Studies, 2, 1960, p. 47-70. Alain Juster, « Le Patriarcat œcuménique, la Grèce, la Turquie », CEMOTI vol. 2-3, 1986, p. 10-19. Samim Akgönul, «Les activités du Patriarcat “œcuménique” du Phanar dans les années 1990 et l'opinion publique turque », CEMOTI, 33, 2002, p. 195-216.

43. Olivier Abel, « Islam et laïcité en Turquie », Migrations et société, 69-70, 2000, p. 105-113.

44. À propos de ce projet et des négociations associées, Iannis Konidaris,

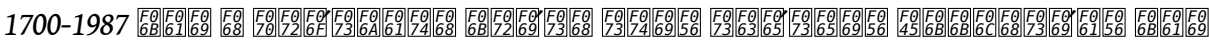

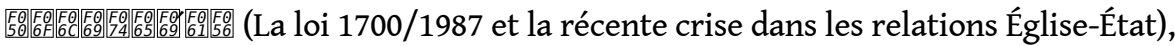
Athènes, Sakkoulas, 1991.

45. En mars 1987, la hiérarchie orthodoxe ordonnait, notamment, au clergé de ne pas célébrer les services liturgiques du 25 mars, jour de la fête nationale et de laisser carillonner les cloches, en signe de deuil.

46. AFP-Athènes, 18/03/1987, 24-26/03/1987. Le Monde, 28/03/1987.

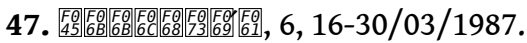

48. La loi 1700/1987, adoptée en avril 1987, ne fut jamais appliquée.

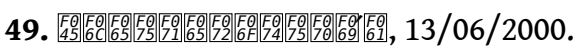

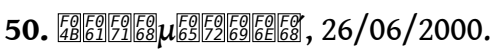

51. Une représentation inscrite dans la légende et dans l'historiographie nationales, symbole d'une révolution sacrée, portée par l'autorité religieuse. Spyros Asdrachas,

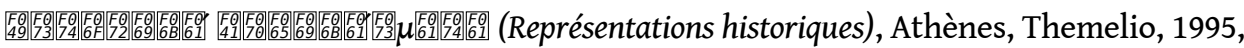
p. 101-104.

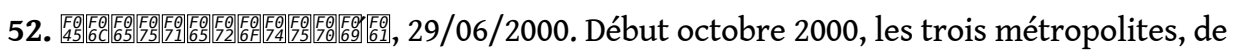
Thèbes, de Kozanis et de Zakynthos sont invités à justifier leur position devant les instances synodales. 


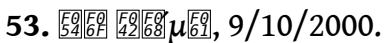

54. doit être replacée dans son contexte polémique.

55. Le Phanar est un quartier d'Istanbul qui abrite, depuis le début du xviie siècle, le siège du Patriarcat grec orthodoxe. Par association métonymique, le terme symbolise, pour l'Église orthodoxe, le Patriarcat de Constantinople.

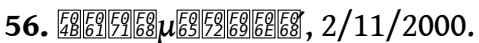

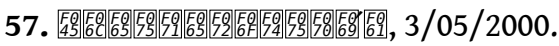

58. Idem.

59. John Petropoulos, Politics and Statescraft in the Kingdom of Greece, Princeton, Princeton University Press, 1968. Douglas DAKIN, The Unification of Greece 1770-1923, Londres, E. Benn, 1972.

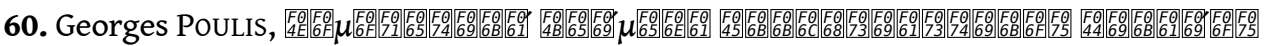
(Textes de droit ecclésiastique), Sakkoulas, Salonique, 1997, p. 550.

61. La notion d'helléno-ottomanisme s'inscrit dans ce débat. Cette formulation identitaire a pu étayer un projet politique, une alternative à l'État nation, dans sa logique mono-

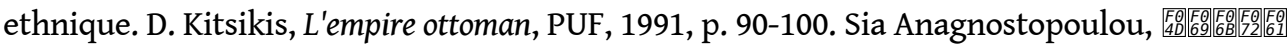

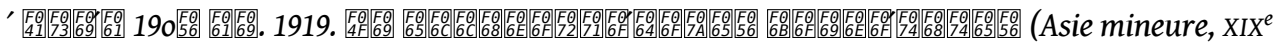
siècle-1919. Les communautés grecques orthodoxes), Athènes, Ellinika Grammata, 1998.

62. Paschalis KITROMILIDIs, « Greek Irredentism in Asia Minor and Cyprus », Middle Eastern Studies, 26, 1990, p. 3-17.

63. Le 8 septembre 1922, les troupes grecques évacuent précipitamment Smyrne. Environ 30000 chrétiens auraient alors péri, dans une ville détruite, quelques jours plus tard, par un incendie. Ce revers militaire aura pour conséquence directe la mort ou le départ précipité de l'essentiel des communautés chrétiennes et/ou grecques d'Asie mineure au XX ${ }^{\mathrm{e}}$ siècle. Michael LLEWELLYN-SMITH, Ionian Vision: Greece in Asia Minor, 1919-1922, Londres, Hurst and Company, 2002 (1973).

64. Alain Wurfbain, L'échange gréco-bulgare des minorités ethniques, Paris, 1930. Perry Lafazani, Myronas Myridis, «L'installation des Grecs du Pont en Macédoine », in Michel Bruneau, dir., Les Grecs Pontiques, Paris, CNRS Éditions, 1998, p. 189-200.

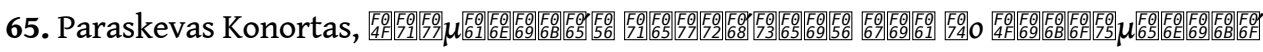

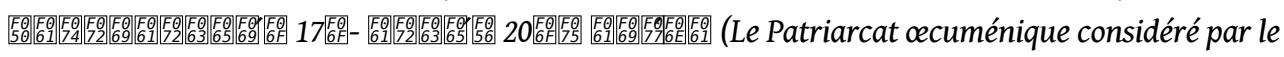
pouvoir ottoman XVII ${ }^{e}$-début du XXe siècle), Athènes, Alexandreia, 1998.

66. À titre d'exemple, lors d'une visite de Mgr Bartholomaios en Grèce, en juin 1999, le métropolite de Salonique souligne, dans son discours d'accueil, qu'il relève « du trône œcuménique ». Des dons financiers au Patriarcat accompagnent ces déclarations. AFP, 6/06/1999.

67. Olivier Clément, Entretiens avec le Patriarche Athinagoras, Paris, Fayard, 1969. Olivier Clément, La vérité vous rendra libre : entretiens avec le Patriarche cecuménique Bartholomée I ${ }^{e r}$, Paris, J.C. Lattès-Desclée de Brouwer, 1996.

68. AFP-Athènes, 4/02/1992.

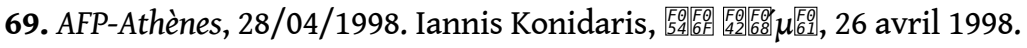

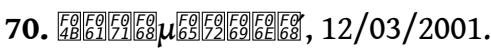

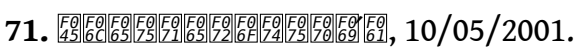

72.

73. Analyse développée par des laïcs et des membres du haut clergé orthodoxe, notamment dans l'entourage de Mgr Christodoulos. 


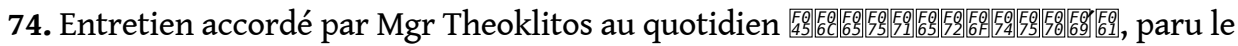
2/05/2001.

75. Pour certains théologiens, le terme même de "primat ", symbole d'une institution centralisée autour d'un seul homme, aurait des accents catholiques romains. Une tradition « étrangère ", donc, qui sert volontiers de repoussoir. Une partie des fidèles et des prêtres de l'Église orthodoxe se rallierait aisément à ce type d'argument.

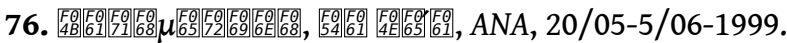

77. Lorsque l'on parle du Patriarcat, il faut préciser que cette petite administration est composée de laïcs ou de prêtres de culture hellénique, volontiers formés en Grèce, mais aussi dans d'autres pays d'Europe ainsi qu'aux États-Unis. Annuaires de l'Église de Grèce,

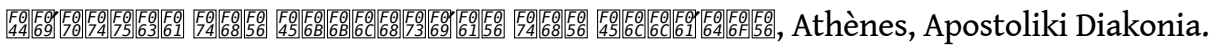

78. Le Patriarcat de Moscou, reconnu autocéphale en 1589, est aboli en 1721. Sa restauration date de 1917 et coïncide avec la chute de la monarchie.

79. Archimandrite Grigorios Papathomas, Le Patriarcat œecuménique de Constantinople (y compris la politeia du Mont Athos) dans l'Europe unie. Approche nomo-canonique, Epektasi, Katerini, 2004 (1998).

80. François Thual, « La mondialisation des religions, toujours recommencée? », Hérodote, 108, 2003, p. 189-205. Pour autant, l'activité, les préoccupations du Patriarche, comme dirigeant religieux incluent bien une dimension territoriale.

81. En Turquie le statut du Patriarche fait l'objet, précisons-le, d'un débat, en l'absence de référence juridique claire. Ainsi, la thèse existe aussi selon laquelle les fonctions internationales du Patriarcat d'Istanbul - par là même reconnues - doivent s'exercer dans le sens des intérêts nationaux de la Turquie.

82. Grigorios Papathomas, « Modalités canoniques de l'exercice de la juridiction du patriarcat œcuménique de Constantinople », Témoignage et Pensée Orthodoxe, 11-12, 1999, p. 1-11.

83. Interlocuteur pour les autres Églises ou communautés religieuses mais aussi pour les responsables politiques de l'Union Européenne, le Patriarche d'Istanbul n'est pas soutenu, de manière privilégiée et structurelle, par un État. Ses ressources proviennent largement de l'extérieur, notamment des communautés grecques orthodoxes de la diaspora, celles d'Amérique en premier lieu. Ces aspects ont des implications, en termes de pouvoir effectif, de marges d'action.

\section{RÉSUMÉS}

Cet article considère les liens complexes de l'Église orthodoxe à l'État, à la nation, au territoire, en Grèce contemporaine et envisage leur possible redéfinition, au seuil du XXI ${ }^{\mathrm{e}}$ siècle. À cet égard, il peut être intéressant de revenir sur l'affrontement théâtralisé qui oppose, dans les dernières années du XXe siècle, l'Église orthodoxe de Grèce au gouvernement de Kostas Simitis, avec, pour point de cristallisation, la suppression de l'indication confessionnelle sur les cartes d'identité. L'opération de résistance impulsée alors par l'Église témoigne, certes, des rapports de cette instance religieuse au pouvoir séculier : l'argument identitaire - ou nationaliste - vient ici consolider le refus de toute séparation entre Église orthodoxe et État. Il faut pourtant se 
demander dans quelle mesure cette mobilisation n'a pas d'abord eu, pour fonction sous-jacente d'affirmer, au sein de l'Église, l'autorité de l'archevêché d'Athènes. La manière dont s'est articulée, dans ce conflit, la figure du Patriarche « œcuménique » ne peut qu'étayer cette lecture. Cette configuration nous renvoie aux étapes fondatrices de l'Église orthodoxe de Grèce, au sein de laquelle deux pôles principaux se distinguent, depuis le milieu du XIX siècle : le Patriarcat orthodoxe de Constantinople/Istanbul et l'évêché d'Athènes. Autour de ces deux pôles se sont noués des enjeux d'autorité, que réactivent, au seuil du XXI ${ }^{\mathrm{e}}$ siècle, les transformations de l'espace européen.

This article deals with the Orthodox Church of Greece and its complicated connections to the State, the nation, the territory. We consider the possible redefinition of these links, at the eve of the $\mathrm{XXI}^{\text {st }}$ century. From this point of view, it may be interesting to go back to the dramatized confrontation which opposed, in 2000-2001, the Orthodox Church of Greece to the socialist government and whose focus point was the suppression of religious affiliation on the identity cards. The resistance initiated by the Church reflects, indeed, the latter's relationship to secular power : the identity argument - or nationalist theme - consolidated, here, the refusal of any separation between the dominant Church and the State. It is, however, possible to read this mobilization first as an expression of internal ecclesiastical stakes. The subtle insertion of the "Ecumenical" Patriarcate in this conflict may buttress this reading. This sends us back to the foundation stages of the Orthodox Church of Greece, within which two main poles emerge after the first half of the XIX ${ }^{\text {th }}$ century : the Orthodox Patriarchate of Constantinople and the Bishopric of Athens. Around these two poles, authority issues have taken shape. The recent transformation of the European space tends to stir up these issues.

Este artículo considera los lazos complejos de la Iglesia ortodoxa con el Estado, con la nación y con el territorio en Grecia contemporánea, y considera su posible redefinición, en el umbral del siglo XXI. En este sentido, puede ser interesante volver sobre el enfrentamiento teatralizado que opone, en los últimos años del siglo XX, a la Iglesia ortodoxa de Grecia con el gobierno de Kostas Simitis, cuyo punto de cristalización es la supresión de la referencia confesional en los documentos de identidad. La operación de resistencia impulsada entonces por la Iglesia da cuenta, ciertamente, de las relaciones de esta instancia religiosa con el poder secular: el argumento identitario - o nacionalista - viene a consolidar el rechazo de toda separación entre Iglesia ortodoxa y Etado. Es necesario, sin embargo, preguntare en qué medida esta movilización no tuvo, en principio, como función subyacente la de afirmar, en el seno de la Iglesia, la autoridad del arzobispo de Atenas. La manera en que se articuló, en esta conflicto, la figura del Patriarca "ecuménico" no puede sino apoyar esta lectura. Esta configuración remite a las etapas fundadoras de la Iglesia ortodoxa de Grecia, en el seno de la cual dos polos principales se distinguen desde mediados del siglo XIX : el patriarcado ortodoxo de Constantinopla/Istambul el obispado de Atenas. Alrededor de esos dos polos se desplegaron disputas de autoridad, que reactivan, en el iumbral de siglo XXI, las transformaciones del espacio europeo.

\section{INDEX}

Mots-clés : Eglise Orthodoxe et Etat, Grèce, identité confessionnelle, Patriarcat orthodoxe 
AUTEUR

ISABELLE DÉPRET

École française d'Athènes 